\title{
Erratum to: Wolbachia-Free Heteropterans Do Not Produce Defensive Chemicals or Alarm Pheromones
}

\author{
Judith X. Becerra ${ }^{1}$ - Gabriela X. Venable ${ }^{2} \cdot$ Vahid Saeidi $^{1}$
}

Published online: 22 September 2015

(C) Springer Science+Business Media New York 2015

\section{Erratum to: J Chem Ecol (2015) 41:593-601}

DOI 10.1007/s10886-015-0596-4

The panels published for Fig. 6 should be in Fig. 5. The legends were published in the correct positions. The real Fig. 6 was missing and is provided below:

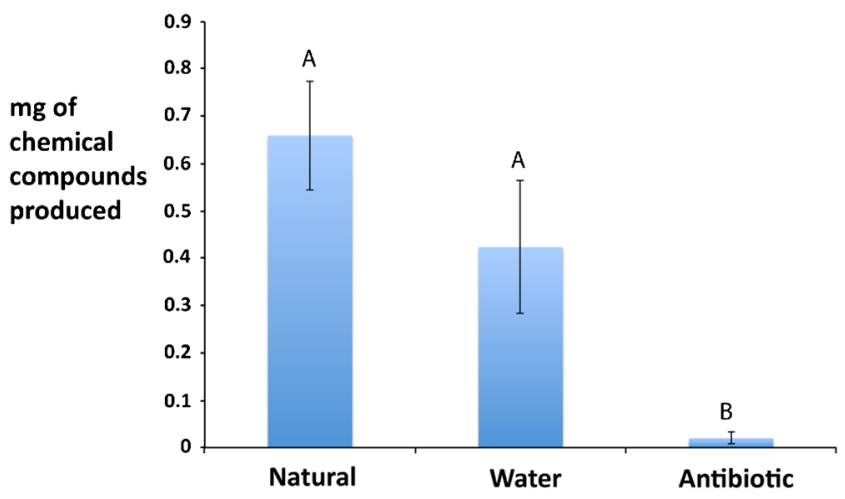

The authors wish to emphasize that they are not claiming that the defensive chemicals and alarm pheromones of all Heteropterans are produced by symbiotic bacteria. They worked with a single species, Thasus neocalifornicus, and it remains to be determined whether the chemical defenses of other Heteropterans also are produced partly or wholly by symbionts.

Fig. 6. Mean production of chemicals by GMB individuals injected with antibiotics was lower than both, not injected (natural), or injected with water $(P<0.0001)$. Same letter above bars indicate no statistical difference between means

The online version of the original article can be found at http://dx.doi.org/ $10.1007 / \mathrm{s} 10886-015-0596-4$

Judith X. Becerra

jxb@email.arizona.edu

Gabriela X. Venable

gxv.gabby.venable@gmail.com

Vahid Saeidi

v.saeidi@gmail.com

1 Department of Biosphere 2, University of Arizona, Tucson, AZ 85721, USA

2 University High School, 421 N. Arcadia Avenue, Tucson, AZ 85711, USA 